\title{
Abdominal Aortic Aneurysm Repair Using the Multilayer Flow Modulator in Porcine Animal Models
}

\author{
Sherif Sultan ${ }^{1,2, *}$, Edel P Kavanagh², Michel Bonneau ${ }^{3}$, Chantal Kang $^{3}$, Niamh Hynes ${ }^{2}$ \\ ${ }^{1}$ Western Vascular Institute, Department of Vascular and Endovascular Surgery, University Hospital Galway, \\ Newcastle Road, Galway, Ireland \\ ${ }^{2}$ Galway Clinic, Doughiska, Galway, Ireland \\ ${ }^{3}$ Centre de Recherche en Imagerie Interventionnelle (CR2i), National Institute of Agronomic Research, Jouy-en-Josas, France \\ *Corresponding Author: sherif.sultan@hse.ie
}

Copyright (C) 2015 Horizon Research Publishing All rights reserved.

\begin{abstract}
This study tested the hypothesis that the Multilayer Flow Modulator (Cardiatis, Isnes, Brussels) can treat aortic aneurysms through intra-arterial hemodynamic modulation, without substantial parent or small branch artery compromise, using 8 porcine models. The animals were euthanized and devices explanted upon completion of the study for examination. Results of the delivery system evaluation showed that there were no difficulties during navigation of the device through the arteries. An overall trend of reduction in aneurysm size was recorded. The opening of the aneurysm was nearly occluded with thrombus in 2 of the explants. The visceral arteries covered by the device remained patent, and it adhered to the arterial wall with endothelialization clearly visible. The MFM was therefore placed without intra-arterial compromise, and the aneurysms were stabilized while adequate blood flow to the collateral arteries was preserved.
\end{abstract}

Keywords Multilayer Flow Modulator, Aneurysm Repair, Porcine Model, Intra-arterial

\section{Introduction}

Total exclusion of the aneurysm sac is common practice with traditional endovascular grafts however in some cases fenestration holes are placed in the grafts to allow for collateral branch flow. [1] Despite such advances in endovascular repair, it has been suggested that, as a challenge inherent to the use of covered stents, total exclusion of the aneurysm may subject the sac to increased pressure and risk of rupture. [2,3] Furthermore, exclusion of the sac often leads to visceral hemodynamic compromise, and inadequate perfusion to peripheral organs, often when collateral arteries feed the aneurysm.

An alternative approach to the management of aortic aneurysms that has recently emerged involves a disruptive technology, in the form of Multilayer Flow Modulator
(MFM) (Cardiatis, Isnes, Brussels). The MFM is a self-expandable mesh of cobalt alloy wires interconnected in five layers (Figure 1). They are extremely flexible with a high kinking and fatigue resistance, and low total porosity. The MFM design physiologically includes the aneurysm by allowing blood to flow through the mesh to maintain collateral branch patency, while modulating turbulent flow to laminar within the device and the aneurysm sac. The laminar flow in the aneurysm reduces shear stress on the aneurysm wall and encourages an organized thrombus formation, thereby stabilizing aneurysm size and reducing risk of rupture. In complex surgical situations, where an open or endovascular repair using a branched or fenestrated graft is the only option, the MFM could represent a viable treatment alternative. Branched and fenestrated graft technologies have not posed any significant advantages over traditional methods. They prove to be considerably more difficult for the operator to utilize, and also require greater pre-operative preparation in terms of artery and aneurysm morphologies. [4] The MFM received Conformité Européenne (CE) marking in Europe in October 2010 for use in aortic aneurysm repair. Many of these devices have been placed in patients on compassionate grounds, but there has been little in vivo documentation of the MFM mechanism of action.

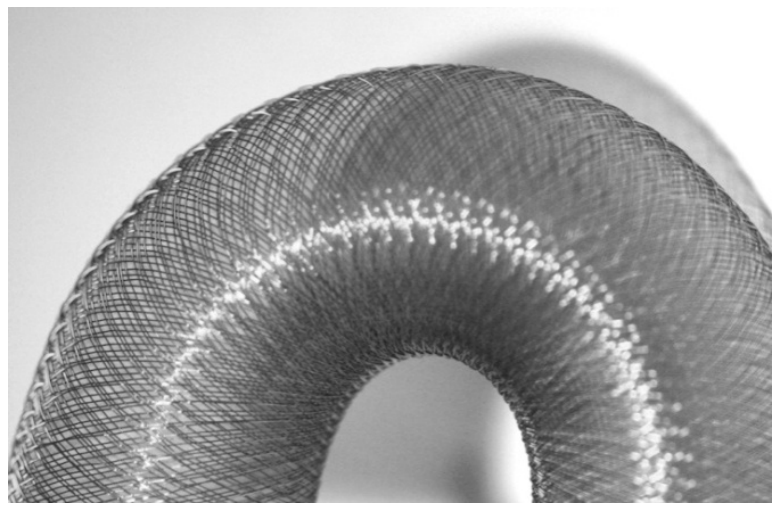

Figure 1. The Multilayer Flow Modulator (MFM) 
Porcine animal models are commonly used as a first-step method of assessment of the safety of installation and functionality of new endovascular technologies. [5-9] Based on a previous study that examined endovascular stent grafts for the prevention of rupture of abdominal aortic aneurysms (AAA) in a sheep model, [5] this study was designed to allow evaluation of the efficaciousness of the MFM in treating induced aneurysms in porcine test animals, without causing substantial parent or small branch artery compromise. Assessment of the expected technical performance of the delivery method was also conducted on a separate animal, which did not have an induced aneurysm.

\section{Materials and Methods}

8 porcine test animals were selected for implantation with the MFM during the study, which was approved and carried out at the Centre de Recherche en Imagerie Interventionnelle (Cr2i) of the National Institute of Agronomic Research (INRA), Jouy-en-Josas, France. Endoprosthesis performance in aneurysm repair and absence of short-term undesired effects was evaluated. A separate porcine test animal was selected for analysis of the delivery system only, evaluating the facility, and reliability of navigability and release of the device without complications.

All of the selected animals came from the Bretoncelles breeding farm (Ferme des Noyers, F-61110, France), and received humane care in accordance with EN IS0 10993-2:2006 Biological Evaluation of Medical DevicesPart 2: Animal Welfare Requirements and Directive 2010/63/EU on the protection of animals used for scientific purposes.

\subsection{Procedure for MFM Delivery System Evaluation}

The delivery release system consisted of a catheter and push rod. The stents were loaded into the catheter at the device manufacturing facility. This evaluation of the delivery system used 1 test animal, a dwarf pig (FBM miniature pig) with a weight of $39 \mathrm{~kg}$ and age 6 months. The delivery system evaluation consisting of 8 separate device deployments was completed in 1 day, with the animal euthanized at the end of the procedure.

The technical success of deployment was evaluated according to the following primary parameters. (1) Successful installation of the implantation system, defined as successful placement of the device in a pre-release position in the artery according to the pinpointed anatomical location. If installation failure occurred, an example of a possible cause could be too great flexibility of the insertion system, which would prevent navigation of the curves encountered in the animal's vascular system. (2) Absence of dissections during installation of the implantation system, confirmed by fluoroscopy during implantation and by visual examination after sacrifice. (3) Successful release of the MFM. (4) Implantation of the MFM at the desired site (precision of positioning), evaluated with fluoroscopy by comparing the position of the deployed MFM with the reference mark placed on the catheter guide tube, and estimated by the operator on a scale of 1 to $3(1=\operatorname{good}, 2=$ average, $3=$ poor $)$. (5) Correct expansion of the MFM. (6) Absence of immediate thrombosis on the device. Secondary parameters evaluated were the ease of installation system handling and placement, and the ease of withdrawal of the release system without damage such as dissection, thrombosis, or rupture.

Table 1. Evaluation of the MFM Delivery System in 1 Pig without Induced Aneurysms

\begin{tabular}{|c|c|c|c|c|c|c|c|c|}
\hline \multicolumn{9}{|c|}{ Delivery Animal } \\
\hline Stent number & 1 & 2 & 3 & 4 & 5 & 6 & 7 & 8 \\
\hline Implanted vessel & $\begin{array}{l}\text { Left } \\
\text { femoral }\end{array}$ & Left iliac & $\begin{array}{c}\text { Left } \\
\text { humeral }\end{array}$ & $\begin{array}{c}\text { Left } \\
\text { axillary }\end{array}$ & $\begin{array}{c}\text { Left } \\
\text { axillary }\end{array}$ & $\begin{array}{c}\text { Left } \\
\text { axillary }\end{array}$ & Aorta & Aorta \\
\hline \multicolumn{9}{|c|}{ Primary Parameters } \\
\hline $\begin{array}{c}\text { Successful } \\
\text { installation of } \\
\text { insertion system }\end{array}$ & Yes & Yes & Yes & Yes & Yes & Yes & Yes & Yes \\
\hline $\begin{array}{l}\text { Dissection during } \\
\text { insertion }\end{array}$ & No & No & No & NA & No & No & No & No \\
\hline Stent release & Yes & Yes & Yes & No & Yes & Yes & Yes & Yes \\
\hline $\begin{array}{l}\text { Precision of } \\
\text { positioning }\end{array}$ & Yes & Yes & Yes & NA & Yes & Yes & Yes & Yes \\
\hline $\begin{array}{c}\text { Correct stent } \\
\text { expansion }\end{array}$ & Yes & Yes & Yes & NA & Yes & Yes & No & Yes \\
\hline $\begin{array}{l}\text { Immediate thrombus } \\
\text { on stent }\end{array}$ & No & No & No & NA & No & No & No & No \\
\hline \multicolumn{9}{|c|}{ Secondary Parameters* } \\
\hline Ease of handling & 3 then 1 & 1 & 1 & $\mathrm{n} / \mathrm{a}$ & 1 & 1 & 1 & 1 \\
\hline Ease of release & 1 & 1 & 1 & 3 & 1 & 1 & 1 & 1 \\
\hline
\end{tabular}

NA: not applicable, $*$ Rated on a 3 -point scale $(1=\operatorname{good}, 2=$ average, $3=$ poor $)$ 
The animal was put on a fast 24 hours before the operation. A premedication of ketamine $(1000 \mathrm{mg})$ was administered intramuscularly. The animal was then intubated and kept under general anesthesia with a mixture of isoflurane $(1.5 \%$ $2 \%)$ and oxygen $(98 \%-98.5 \%)$ throughout the operation. A physiological follow-up (oximetry, pulse, and heart rate) was provided during the entire operating procedure. A total of 8 MFM devices were deployed through right femoral access (Seldinger technique), [10] with a short guide tube (7-F) employed in first attempts, and a 7-F bearing catheter (Arrow International, Reading, PA, USA) used for a left contralateral implantation (left femoral and iliac arteries) on the second attempts with device 1 and device 2. For the remaining implanted devices, the short guide tube was repositioned. The arterial sites for implantation of the 8 MFM devices are shown in Table 1. When all 8 implantation procedures had been completed, the animal was sacrificed by lethal barbiturate intravenous injection.

\subsection{Procedure for Evaluation of MFM Performance in Induced Aneurysms}

In accordance with the procedures in previously conducted animal-model device studies, $[5,6] 8$ dwarf pigs (average weight $50 \mathrm{~kg}$ ) were prepared 24 hours before MFM implantation. The surgical creation of the induced pseudo-aneurysm was performed under general anesthesia. By way of a neck incision, an external jugular vein was harvested. Upon good hemostasis, closure of the neck wound was conducted. An incision was then made in the left flank of the animal, with dissection and surgical exposure of the abdominal aorta through the retroperitoneal structures. Clopidogrel $(5 \mathrm{mg} / \mathrm{kg}$ ) and unfractionated heparin $(70 \mathrm{IU} / \mathrm{kg})$ were administered by intravenous injection before aortic clamping. Following clamping, grafting of the vein segment was carried out with a side-to-side anastomosis to form a saccular aneurysm in 6 of the animals (Figure 2A), and with an interposition graft to form a fusiform aneurysm in the remaining 2 animals (Figure 2B). Then followed declamping, establishment of hemostasis, and restoration of arterial pressure to the aneurysm.

For the MFM implantation, arterial access was gained through the femoral artery using the Seldinger technique, [10] and the device was deployed in the abdominal aorta, inferior to the level of the renal arteries. Angiography was performed to monitor the aneurysms. Measurements of the diameter of the artery, the diameter and size of the aneurysm, and blood pressure at the level of the aneurysmal sac were tracked by angiography throughout the duration of the surgery. The devices were implanted across the necks of the vein-graft induced aneurysms. Follow-up with angiography was scheduled for 1 week post-implantation, and explantation was planned for 30 days. After angiographic checking at the end of the study period, the animals were euthanized with a Doléthal intravenous injection $(100 \mathrm{mg} / \mathrm{kg})$. The stents and aneurysms were explanted and examined, and macroscopic images recorded.
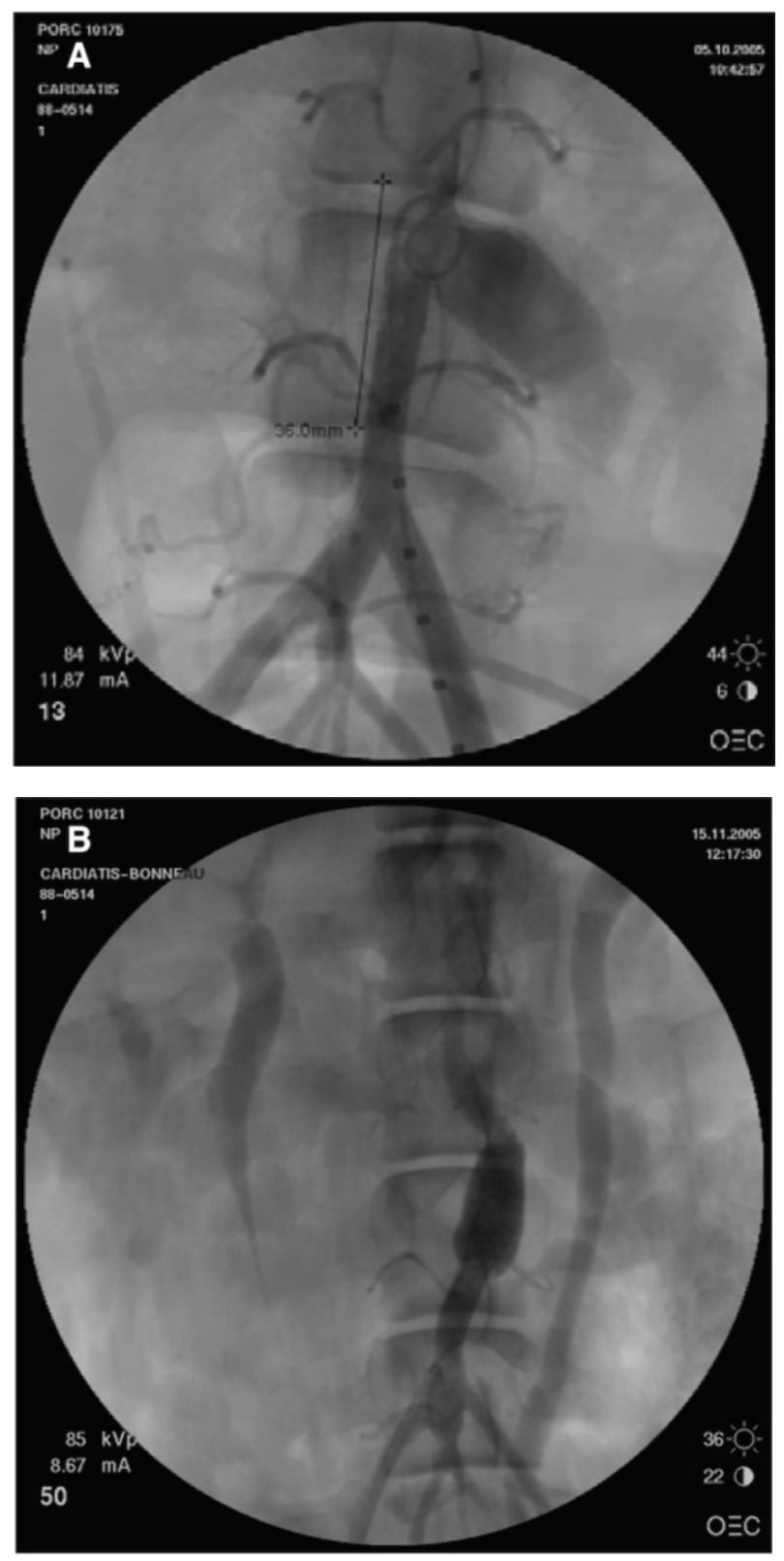

Figure 2. Aneurysm angiograms: (A) saccular, (B) fusiform

The MFM performance was evaluated according to the following primary parameters. (1) Absence of dissection during device implantation, confirmed by fluoroscopy during implantation and by visual examination after sacrifice. (2) Proper expansion of the device, assessed by the operator on a scale of 1 to 3 ( 1 = easy, 2 = difficult, 3 =impossible). (3) Absence of immediate thrombosis on the device, as monitored by arteriography. (4) Aneurysm exclusion, monitored by angiography at various time points after device implantation. (5) Absence of stenosis on the device, as monitored by arteriography. (6) Endothelialization of the inner surface of the device, assessed macroscopically after sacrifice on a scale of 1 to $3(1=$ absence of lining, $2=$ partial lining, 3 = total lining). (7) Absence of pathology on explanted devices, assessed by histology.

Secondary parameters evaluated were successful release 
and positioning of the device, monitored by fluoroscopy and assessed by the operator on a scale of 1 to $3(1=$ good, neck of aneurysm totally covered by the device; $2=$ medium, aneurysm partially covered, leaving less than $10 \%$ passage; 3 $=$ bad, ill-positioned device covering the aneurysm neck on less than $90 \%$ of surface), and absence of device migration, by comparison of post-implantation and pre-explantation radiographic images.

\section{Results}

\subsection{MFM Delivery System Evaluation}

Table 1 shows the results that were recorded during the MFM delivery system evaluation, with 8 devices implanted separately in one porcine test animal. It was not possible to implant device 1 and device 2 in the first attempt by crossing over the stents in the left femoral and left iliac arteries. However, the use of a bearing catheter (Arrow type 7-F) made it possible to perform a contralateral implantation on the second attempt in each case. No difficulty was encountered in advancing the catheter carrying the device when the angulation at the iliac bifurcation was calculated at $36^{\circ}$. Both stents were positioned correctly and expanded without complication, and no immediate thrombus of the devices was recorded. For devices 3, 4, 5 and 6, navigating to the implantation site (left humeral artery for device 3, left axillary artery for devices 4,5 , and 6 ) was carried out along a 0.025 -inch exchanger guide. Strong resistance was noted on the push rod for device 3 at the beginning of release, but there was no difficulty positioning the MFM during its release in the humeral artery. Device 4 could not be released because the push rod used for the release was not long enough. This issue was rectified before device 5 and device 6 were implanted. Device 5 was deployed without complication, and device 6 , which was overlapped by 2 of the already implanted devices, behaved normally. With the exception of device 4 , each of the devices were positioned and expanded without complication, and no immediate device thrombus was recorded. Devices 7 and 8 were implanted in the aorta. Device 7 was not completely deployed in the distal portion; device 8 was positioned such that it partly superimposed on device 7 , but its deployment occurred normally, and again, no immediate thrombus was recorded.

\subsection{Evaluation of MFM Performance in Induced Aneurysms}

\subsubsection{Aneurysm Formation}

All pseudo-aneurysms were prepared at the level of infrarenal abdominal aorta. The average size of the normal aortic vessel was $8.5 \pm 2.2 \mathrm{~mm}$ (range 6.5 to $13.1 \mathrm{~mm}$ ) (Table 2). Saccular aneurysms were induced in 6 pig models (animals A to F), while fusiform aneurysms were induced in 2 pig models (animals $G$ to $H$ ). The aneurysms varied in size, depending on the size of the jugular vein available for grafting. The average aneurysm diameter was $22.2 \pm 7 \mathrm{~mm}$ (range 13.1 to $31.1 \mathrm{~mm}$ ) and the mean aneurysm length was $23.2 \pm 13.5 \mathrm{~mm}$ (range 11.2 to $50.9 \mathrm{~mm}$ ). The diameter of each of the 2 fusiform aneurysms ( $13.1 \mathrm{~mm}$ for animal $\mathrm{G}$ and $18.4 \mathrm{~mm}$ for animal $\mathrm{H}$ ) was more than twice the diameter of the normal aorta $(6.6 \mathrm{~mm}$ for animal $\mathrm{G}$ and $7.2 \mathrm{~mm}$ for animal $\mathrm{H})$.

Table 2. Evaluation of MFM Performance in 8 Pigs with Experimentally Induced Aortic Aneurysms

\begin{tabular}{|c|c|c|c|c|c|c|c|c|}
\hline \multicolumn{9}{|c|}{ Test Animal } \\
\hline & A & $\mathrm{B}$ & $\mathrm{C}$ & $\mathrm{D}$ & E & $\mathrm{F}$ & G & $\mathrm{H}$ \\
\hline \multicolumn{9}{|c|}{ Stent Features } \\
\hline Diameter/length (mm) & $10 / 50$ & $10 / 50$ & $9 / 40$ & $10 / 40$ & $8 / 50$ & $\begin{array}{l}12 / 50 \\
12 / 40\end{array}$ & $\begin{array}{l}8 / 50 \\
8 / 40\end{array}$ & $\begin{array}{l}12 / 50 \\
12 / 60\end{array}$ \\
\hline \multicolumn{9}{|c|}{ Aneurysm Features } \\
\hline Type & Saccular & Saccular & Saccular & Saccular & Saccular & Saccular & Fusiform & Fusiform \\
\hline Normal vessel diameter (mm) & 13.1 & 7.6 & 8.4 & 9.2 & 6.5 & 9.5 & 6.6 & 7.2 \\
\hline Aneurysm diameter $(\mathrm{mm})$ & 30.1 & 17.1 & 16.1 & 31.1 & 28.4 & 23.4 & 13.1 & 18.4 \\
\hline Aneurysm length (mm) & 28.7 & 14.9 & 11.2 & 18.4 & 14.1 & 14.9 & 32.7 & 50.9 \\
\hline Neck size $(\mathrm{mm})$ & 10 & 10.5 & 6.6 & - & - & - & - & - \\
\hline Blood pressure (max/min) & $156 / 103$ & - & $130 / 83$ & $93 / 60$ & $115 / 76$ & $126 / 83$ & - & - \\
\hline \multicolumn{9}{|c|}{ Primary Efficacy Parameters } \\
\hline Dissection while inserting & No & No & No & No & No & No & No & No \\
\hline Stent release* & 1 & 1 & 1 & 2 & 1 & 2 & 2 & 3 \\
\hline Thrombosis in vascular lumen & No & No & No & No & No & No & No & No \\
\hline Exclusion of aneurysm intraoperatively & No & No & No & No & No & No & No & No \\
\hline Exclusion of aneurysm at 30 days & Yes & Yes & Yes & $\mathrm{n} / \mathrm{a}$ & Yes & $\begin{array}{c}\text { No } \\
(\sim 90 \%)\end{array}$ & Yes & $\mathrm{n} / \mathrm{a}$ \\
\hline \multicolumn{9}{|c|}{ Secondary Efficacy Parameters } \\
\hline $\begin{array}{l}\text { Successful release, positioning at desired } \\
\text { area** }\end{array}$ & 2 & 1 & 1 & 3 & 1 & 2 & 2 & 3 \\
\hline
\end{tabular}

*Rated on a 3-point scale $(1=$ easy, $2=$ difficult, and $3=$ impossible $), * *$ Rated on a 3 -point scale $\quad(1=$ good, $2=$ medium, $3=$ bad $)$ 


\subsubsection{Device Insertion}

Blood pressure measured before placing the stents was high in 4 animals while 1 animal had a normal blood pressure (Table 2). Blood pressure was not recorded for animals B, G and $\mathrm{H}$. Two of the 8 pigs died of ruptured aneurysms: animal $\mathrm{D}$ (saccular aneurysm) at 24 hours, animal $\mathrm{H}$ (fusiform aneurysm) at 3 days. The process of device implantation had been halted in these 2 animals due to difficulty in positioning and releasing the MFM at the required spot (mismatch of the release mechanism with the aneurysm resulting in inadequate coverage of the aneurysm neck). No stent migration occurred upon device insertion in the other 6 animals.

\subsubsection{Angiographic Outcomes}

Immediate post implantation angiographies showed that in none of the test animals was the aneurysm totally excluded after the device had been placed (Table 2), although the angiographies did show that in most cases there was reduced inflow of contrast agent in the aneurysmal sac. At 1-week angiographic follow-up, an overall reduction in aneurysm size was recorded for 3 animals (animal A, B, and E). Figure 3 shows the progressive reduction in aneurysm size for animal $\mathrm{E}$, based on angiographic images after MFM implantation, at 1 -week follow-up, and at 1 month. At 1 -week, animal $\mathrm{C}$ showed total thrombosis of the aneurysm sac. For animal G, 1-week angiography showed that the size of the aneurysm sac remained the same since stent implantation. Animal $F$ had no follow-up at 1-week post-implantation. Upon completion of the study after 30 days, most animals that survived had a totally excluded aneurysm, while in one animal (animal F), the aneurysm was $90 \%$ excluded.

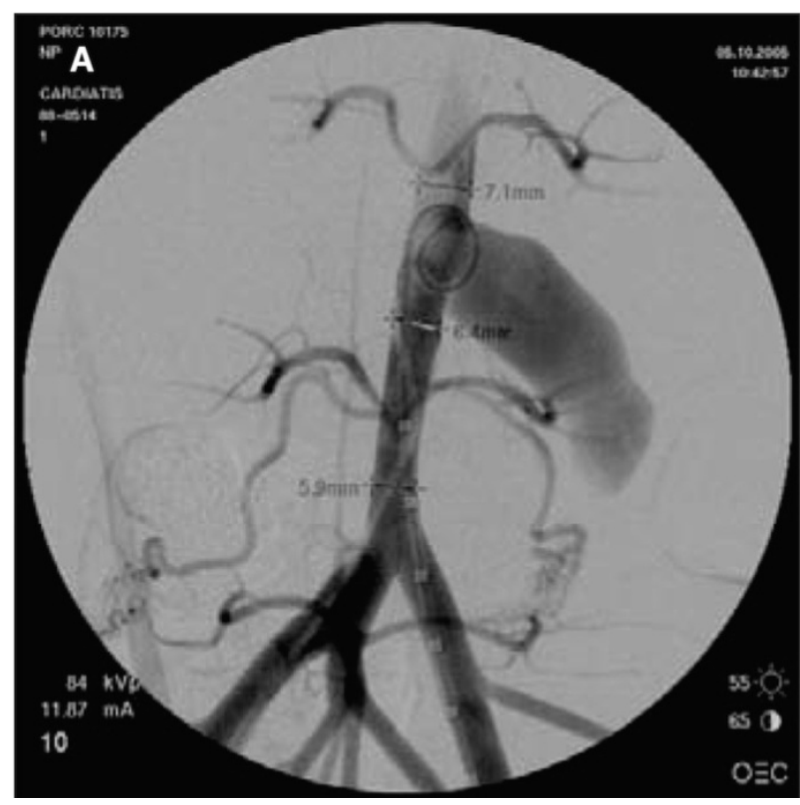

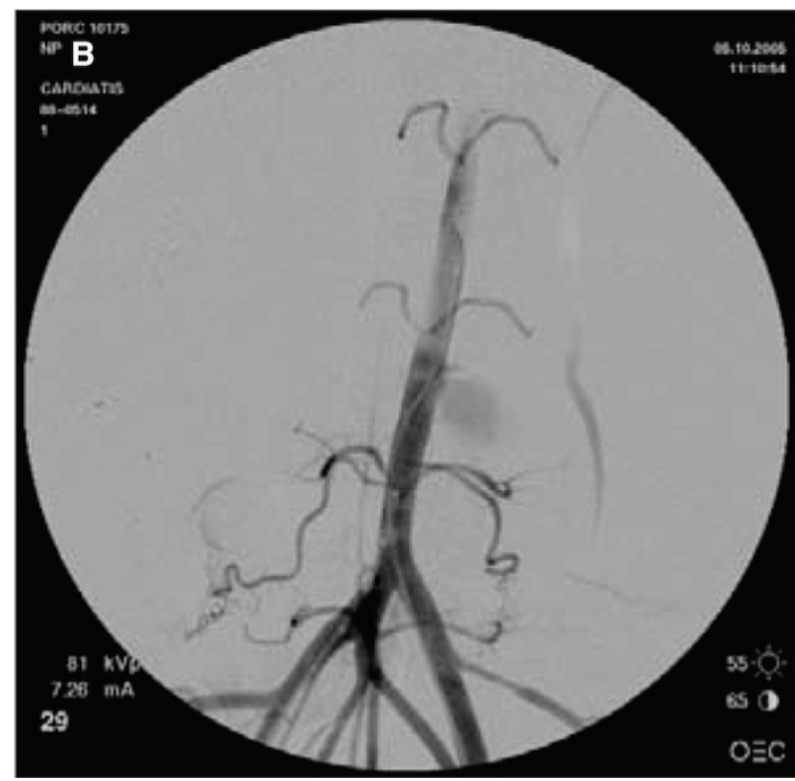

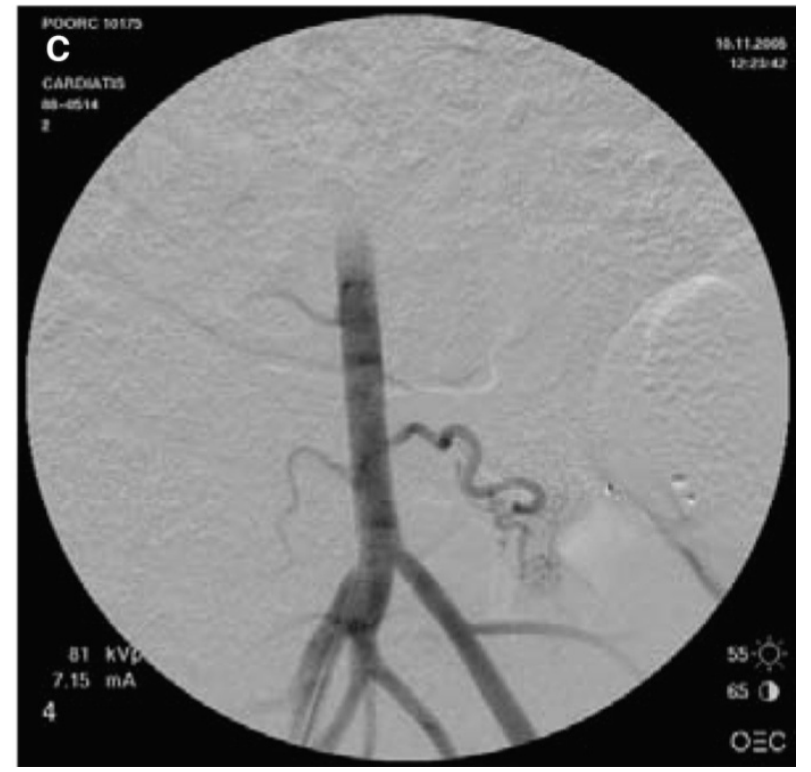

Figure 3. Angiographic images of animal E: (A) implantation; (B) 1 week; (C) 1 month

\subsubsection{Device Explantation}

Most of the animals were planned to be sacrificed at the end of the study (30 days). One animal (animal A) was studied for a shorter period of 10 days. Animal D (saccular aneurysm) and Animal H (fusiform aneurysm) died at 24 hours and 3 days post implantation respectively, due to aneurysm rupture. For animal $\mathrm{D}$, the cause of this rupture could not be explained. For animal $\mathrm{H}$, rupture of its aneurysm seemed to be induced by the dissociation of one wire of the device. No stent migration occurred upon device explantation. In animal A, sacrificed at 10 days, it was evident upon explantation that the stent was not well positioned and particularly did not cover the proximal neck of the aneurysm (Figure 4). Nevertheless the aneurysm in animal A had thrombosed progressively, the device adhered to the parietal wall in a homogeneous manner, and no stenosis was observed in the device. 


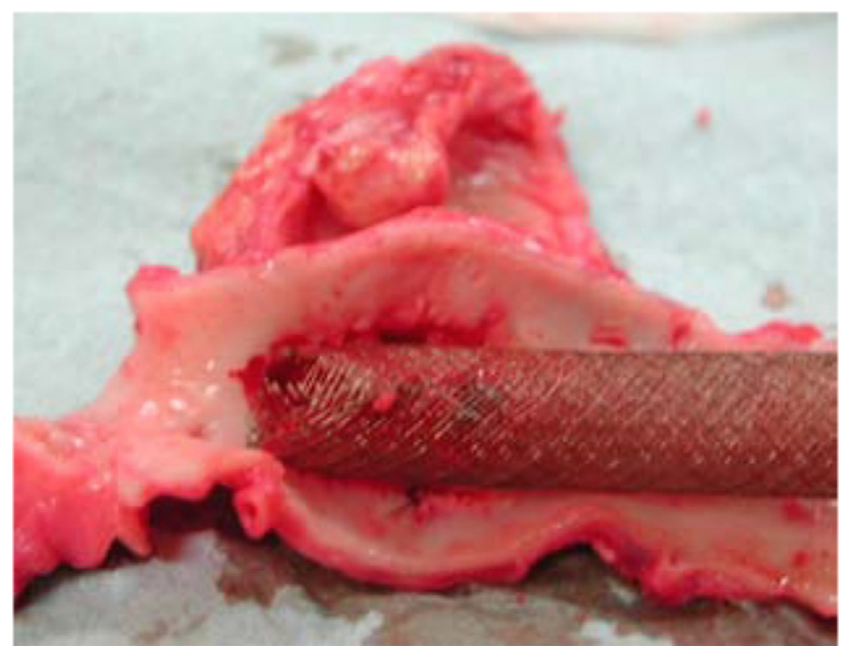

Figure 4. The uncovered proximal aneurysm neck of animal A

\subsubsection{Macroscopic Examination}

In the animals sacrificed at 1 month after implantation (animals B, C, E, F), the evolution of the aneurysms was in favor of a decrease in size. In animal B, the entrance to the aneurysm was occluded by thrombus. The aneurysm of animal $\mathrm{G}$ was assessed for a period of 74 days, explanted, and observed in detail. The parietal wall of the aneurysm no longer presented as a venous wall as its thickness was strongly increased suggesting an evolution to an arterial type wall (Figure 5).

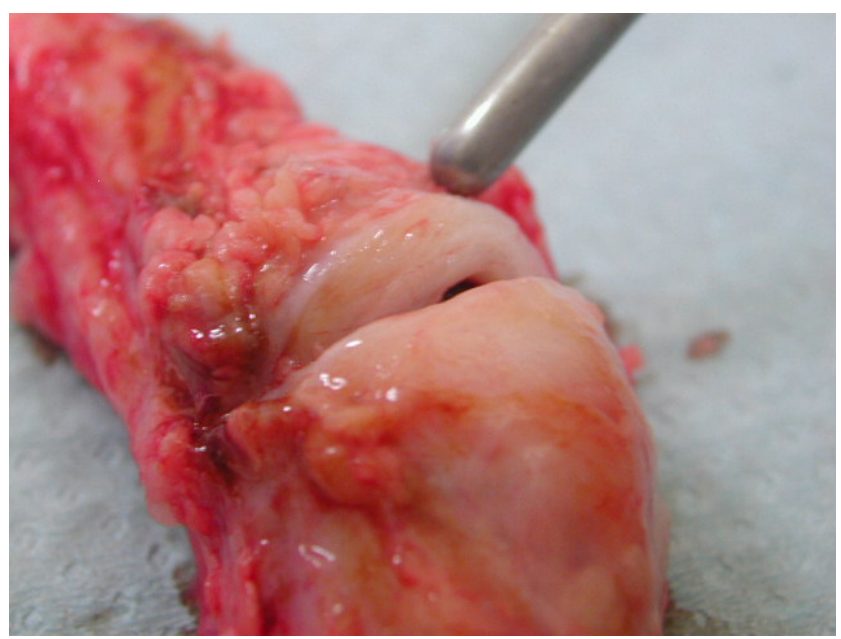

Figure 5. Parietal wall thickening in animal $\mathrm{A}$

\subsubsection{Microscopic Examination}

The evolution to an arterial type wall was also evident during histological examination of animal G's explants. The stent also adhered perfectly to the arterial wall with endothelization clearly visible under electron microscopy (Figure 6A). The visceral arteries covered by the stent remained patent, again visible under electron microscopy (Figure 6B). A total absence of intimal hyperplasia was evident in all explants.
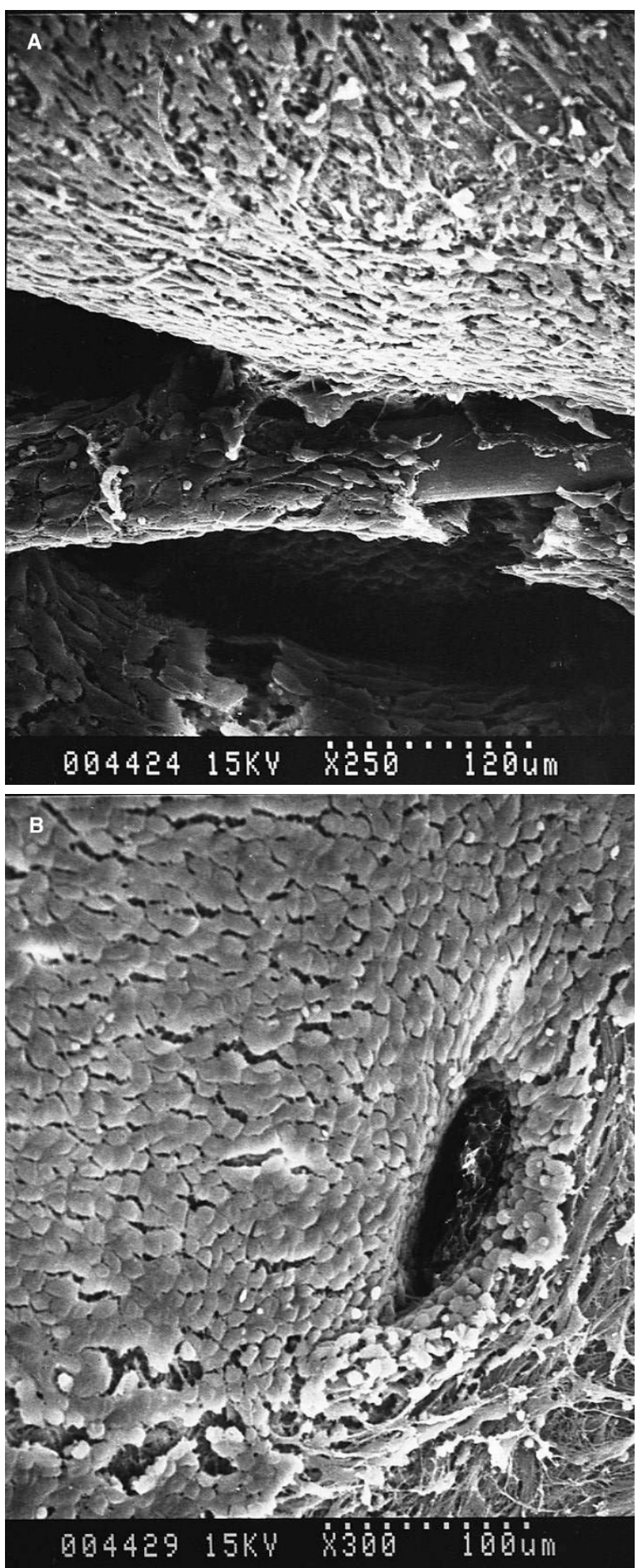

Figure 6. Images of animal $\mathrm{G}$ taken with a scanning electronic microscope: (A) stent endothelialization; (B) vertebral artery patency

\section{Discussion}

This study evaluated the performance of the MFM in 8 porcine test animals, with aneurysms experimentally induced by grafting of venous tissue onto the abdominal aorta. It also evaluated the device delivery system through planned deployment of 8 devices in 1 porcine test animal, without an 
induced aneurysm. In the evaluation of the device installation, there were no complications during navigation of the delivery system through the arteries, positioning of the release mechanism and device deployment were precisely executed, withdrawal of the mechanism occurred without difficulty, and there was a complete absence of immediate complications such as dissection, thrombosis, or rupture. In the evaluation of MFM performance, device installation was successful in 6 of 8 animals, and through the study period in those 6 animals, the vein-graft-induced aneurysms were stabilized, good blood flow was permitted to collateral arteries, and rupture, device migration, and stenosis were completely avoided.

This study, with its positive short-term outcomes in treating aortic aneurysms, provides support for the device concept of aneurysm inclusion rather than exclusion, as the process of aortic remodeling was evident by the formation of thrombus within the aneurysm sac in every model.

\subsection{Animal Models}

Porcine animal models are commonly used as a first step method of assessment of the safety of installation and functionality of new endovascular technologies. [5-9] Diaz et al, [7] developed an in vivo porcine model of a type II endoleak resulting from endovascular aortic aneurysm repair, for the study and treatment of type II endoleak. 8 pigs underwent creation of an infrarenal aortic aneurysm, with a Dacron patch, and after 1 week, the animals underwent EVAR. All 8 animals underwent successful creation of an aortic aneurysm resulting in exclusion of the aneurysm sac. Postoperative computed tomography (CT) scans demonstrated a type II endoleak from the lumbar branches in all animals.

Eckhouse et al, [8] induced descending thoracic aortic aneurysms (TAA) in Yorkshire pigs through intra-adventitial injections of collagenase and periadventitial application of crystalline $\mathrm{CaCl}_{2}$. 3 weeks after TAA induction, the aortas were harvested and tissue collected for biochemical and histological measurements. A subset of animals underwent MRI preoperatively and at terminal surgery. Results demonstrated aortic dilatation, aortic medial degeneration, and alterations in matrix metalloproteinase/tissue inhibitors of metalloproteinase abundance, consistent with TAA formation.

Suk et al, [9] used 6 experimental pigs to simulate ruptured AAA with bleeding and increased abdominal pressure for 4 hours, followed by 2 hours of infrarenal clamping with retransfusion of the lost blood. The bleeding caused a decrease in cardiac output and regional blood flow. An increase of approximately threefold in cytokines, interleukins, and oxidative stress markers was observed. This near-lethal model of ruptured AAA induced expected severe deterioration of hemodynamics and metabolism accompanying a moderate inflammatory and oxidative stress response.
Our study was modeled on that of Boudghene et al, [5] which examined endovascular stent grafts for the prevention of rupture in 16 sheep AAA models, which were created by the insertion of a left internal jugular vein segment that was anastomosed end-to-end to the sectioned infrarenal abdominal aorta. The pseudo-aneurysms in our study were also successfully prepared from a jugular vein section and implanted on the aorta. In addition, angiograms, macroscopic examinations, and microscopic examinations were carried out at various time periods to assess the overall incorporation of the prosthesis into the artery of interest. Across all of these referenced animal studies, the appropriate time of sacrifice was not established based on any findings. In our study, determination of the duration over which useful data could be retrieved with angiographic check-up (1 week follow-up and explantation at 1 month, except for the extended assessment of 1 animal to 2.5 months) was based on the trial and error of the previous animal studies. [5-9] These animal studies from the literature using this model makes this work a coherent addition, and appropriate for translational research.

\subsection{The MFM}

This study directly tested the hypothesis that the MFM stent could treat saccular and fusiform aortic aneurysms through intra-arterial hemodynamic modulation, without substantial parent or small branch artery compromise. Our results showed that the aneurysms were stabilized, since there was no increase in their size over the duration of the study. Examination under electron microscope revealed that adequate blood flow to the collateral arteries was preserved, and there was no thrombus formation at the neck of the artery, leading to the conclusion that organ perfusion was still achievable. This was made possible by the relative permeability of the MFM compared to a traditional covered stent graft.

Explant examination also demonstrated the lack of stenosis on the device or arterial wall, indicating that the MFM placement did not elicit a negative intra-arterial response in the parent artery. The 2 cases of aneurysm rupture demonstrated the importance of model capability.

Great care and experience must be taken when engaging in this technology for the first time so as not to increase vascular injury. The cases of rupture also illustrated the importance of stent positioning; for example, the stent must cover the aneurysm neck completely, a requirement that has been supported by our own clinical experiences with the device. [11]

\subsection{Clinical Outcomes}

To date, the MFM has already been successfully translated to human research. [2,11-16] Many of these early cases were implanted in patients on a compassionate basis due to multiple co-morbidities. Irrespectively, when conducted 
strictly in accordance with the device instructions for use (IFU), deployment of the MFM has produced consistently positive outcomes in aortic pathologies, including TAAA and aortic type B dissections. [2,11-16] Studies reported successful treatment of the aneurysms by shrinkage of the aneurysm, lumen volume reduction and increased formation of an organized thrombus. $[2,11,13,15,16]$ A clinical case by Chocron et al [13] on MFM treatment of thoracoabdominal type B dissections found that a thoracic false lumen was no longer patent 3 months post implantation. To date the MFM has been supported by low rates of perioperative mortality, paraplegia, cerebrovascular accident, and visceral or renal compromise. [11]

Recent studies by Vaislic et al [14] and Debing et al [15] prospectively examined the MFM when used to treat complex aortic aneurysms. These patients were unsuitable for open surgery or endovascular repair with standard, fenestrated or branched grafts. Vaislic et al monitored 23 patients for a duration of 1 year, recording rate of aneurysm thrombosis, branch vessel patency, and all-cause mortality. The rate of technical success was $100 \%$. The rate of primary patency of covered branch vessels was $96 \%$ (53/55). At 12 months, aneurysm diameter was stable in 18 of 20 patients. The mean ratio of thrombus volume to total lumen volume had increased by $21.3 \%(n=17)$. There were no cases of device migration, loss of device integrity, spinal cord ischemia, or aneurysm rupture. Similarly, Debing et al evaluated a smaller cohort of six patients for a duration of 10 months. Four aneurysms were completely thrombosed between 1 and 6 months. The patency of the covered aortic branches was $100 \%$, and at 6 months, the sac volume was decreased in two patients, increased in two patients and remained stable in one patient. There were no stent migrations, retractions, thrombosis, fractures, or reinterventions.

When the MFM is used in scenarios outside of its indications for use, the efficaciousness of the MFM is considerably reduced. [17-21] Therefore, the use of this new technology requires the operator to have a keen knowledge of the device and its appropriate clinical applications before its utilization. The MFM is not limited to aortic pathologies but has also been used to treat peripheral $[22,23]$ and visceral artery aneurysms, [17,22,24-31] again with positive outcomes under the IFU.

There is paucity of long-term data in relation to this new device, with current literature reporting relatively short follow up results. Follow up varies from 1 years in the STRATO trial, conducted by Vaislic et al [14], to 2 years by Balderi et al [25] who evaluated the MFM when used in visceral artery aneurysm repair in 5 patients. While Polydorou et al [22] examined the safety and efficacy of the MFM in patients with aneurysms in different target vessels for a duration of 3 years. The long-term outcomes of the MFM will become clearer with its continued use, and forthcoming publication of the 2-year STRATO results, but initial study results are promising.

\section{Study Limitations}

The study is limited by the animal aneurysm model, which was constructed from venous tissue and prepared 24 hours prior to stent intervention, whereas a human aneurysm forms in the wall of the artery, often over a number of months or years. The use of a young and otherwise healthy porcine test animals limited examination of the delivery system. Their arteries are not comparable to the often tortuous and/or calcified vessels found in older human patients. The study is also limited by its design, in that animal studies often automatically control many variables, which are beyond control in normal human surgical situation. [32] Animal studies therefore do not include naturally occurring variation. [33]

\section{Conclusions}

In this evaluation of the performance of the MFM in a porcine animal model, in the 6 of the 8 cases in which implantation of the device was successful, the vein-graft-induced aneurysms were stabilized while good blood flow was permitted and rupture was avoided. Within the limited scope of the study, the device design principle was confirmed by the observation of thrombus formation in association with maintenance of collateral branch patency and avoidance of device-related stenosis. In the separate evaluation of the MFM delivery system in a single animal, all 8 study devices were delivered to their respective implantation sites without intra-arterial compromise, and 7 of the 8 were successfully deployed.

The study outcomes underscored the importance of proceeding according to the device IFU in terms, for example, of anatomical case planning and execution of delivery mechanics. Further studies are needed, with a larger cohort to complete assessment of the long-term safety and efficaciousness of the MFM. These studies are central to increasing the scientific knowledge surrounding the mechanism of action of the MFM, and as a basis for continuous translational research, such as clinical cases of aneurysm repair and arterial pathologies.

\section{Acknowledgements}

This study was sponsored by Cardiatis (Isnes, Belgium).

\section{REFERENCES}

[1] C. Lioupis, M.M. Corriveau, KS. Mackenzie, D.I. Obrand, O.K. Steinmetz, K. Ivancev, C.Z Abraham. Paraplegia prevention branches: a new adjunct for preventing or treating spinal cord injury after endovascular repair of thoracoabdominal aneurysms, Journal of Vascular Surgery,Vol. 54, No. 1,252-257, 2011. 
[2] S. Sultan, N. Hynes. Disruptive endovascular technology with Multilayer Flow Modulator stents as a therapeutic option in the management of thoracoabdominal aortic aneurysms. Early results from the global independent MFM Registry,Italian Journal of Vascular \& Endovascular Surgery,Vol. 19,No. 4, 215-218,2012.

[3] W. Euringer, M. Südkamp, B. Rylski, P. Blanke. Endovascular Treatment of Multiple HIV-related Aneurysms Using Multilayer Stents.Cardiovascular \& Interventional Radiology, Vol. 35, No. 4,945-949, 2012.

[4] I.M.Nordon, R.J.Hinchliffe, B.Manning, K.Ivancev, P.J. Holt, I.M. Loftus. M.M. Thompson. Toward an "off-the-shelf" fenestrated endograft for management of short-necked abdominal aortic aneurysms: an analysis of current graft morphological diversity,Journal of Endovascular Therapy,Vol. 17, No. 1, 78-85, 2010.

[5] F.P. Boudghene, M.R. Sapoval, M. Bonneau, A.F. LeBlanche, F.C. Lavaste, J.B. Michel. Abdominal aortic aneurysms in sheep: prevention of rupture with endoluminal stent-grafts,Radiology,Vol. 206, No. 2,447-454,1998.

[6] P. Gouny, C. Hocquet-Cheynel, C. Martin-Mondiere, J. Bensenane, M. Bonneau, O. Nussaume. Incorporation of fibronectin-impregnated vascular prostheses in the pig. Microscope study,Journal of Cardiovascular Surgery,Vol. 36, No. 6,573-580,1995.

[7] S.Diaz, M.R.Uzieblo, K.M.Desai, M.R. Talcott, K.T. Bae, P.J. Geraghty, J.C. Parodi, G.A. Sicard, L.A. Sanchez, E.T. Choi. Type II endoleak in porcine model of abdominal aortic aneurysm,Journalof Vascular Surgery,Vol.40, No. 2,339-344,2004.

[8] S.R.Eckhouse, C.B.Logdon,J.M.Oelsen,R.K. Patel, A.D. Rice, R.E. Stroud, W.B. Wince, R. Mukherjee, F.G. Spinale, J.S. Ikonomidis, J.A. Jones. Reproducible porcine model of thoracic aortic aneurysm,Circulation,Vol. 128, No. 11 Suppl 1,S186-193,2013.

[9] P.Suk, I.Cundrle Jr., J.Hruda, L. Vocilková, Z. Konecny, M. Vlasin, M. Matejovic, M. Pavlik, V. Zvoníček, V. Sramek. Porcine model of ruptured abdominal aortic aneurysm repair,EuropeanJournal of Vascular \& Endovascular Surgery,Vol. 43, No.6 ,698-704,2012.

[10] Z.C.Higgs, D.A.Macafee, B.D.Braithwaite, C.A. Maxwell-Armstrong. The Seldinger technique: 50 years on, The Lancet,Vol. 366, No. 9494,1407-1409, 2005.

[11] S. Sultan, N. Hynes. One-year results of the multilayer flow modulator stent in the management of thoracoabdominal aortic aneurysms and type $\mathrm{B}$ dissections, Journal of Endovascular Therapy,Vol. 20,No. 3, 366-377,2013.

[12] S. Sultan, M.Sultan, N.Hynes. Early mid-term results of the first 103 cases of multilayer flow modulator stent done under indication for use in the management of thoracoabdominal aortic pathology from the independent global MFM registry,Journal of Cardiovascular Surgery,Vol.55, No. $1,21-32,2014$

[13] S. Chocron, C. Vaislic, D. Kaili, J.F.Bonneville. Multilayer stents in the treatment of thoraco-abdominal residual type B dissection,Interactive Cardiovascular \& Thoracic Surgery, Vol. 12, No. 6,1057-1059,2011.

[14] C. Vaislic, J.Fabiani, S.Chocron, J. Robin, V.S. Costache, J.P.
Villemot, J.M. Alsac, P.N. Leprince, T. Unterseeh, E. Portocarrero,Y. Glock, H. Rousseau, STRATO Investigators Group. One-year outcomes following repair of thoracoabdominal aneurysms with the Multilayer Flow Modulator: report from the STRATO trial, Journal of Endovascular Therapy,Vol. 21, No. 1, 85-95,2014.

[15] E. Debing, D. Aerden, S. Gallala, F. Vandenbroucke, P. Van den Brande. Stenting Complex Aorta Aneurysms with the Cardiatis Multilayer Flow Modulator: First Impressions, European Journal of Vascular \& Endovascular Surgery, Vol. 47, No. 6, 604-608,2014.

[16] V.S. Tolva, P.G. Bianchi, L.V. Cireni, A. Lombardo, G.C. Keller, G. Parati, R.M. Casana. Multiple multilayer stents for thoracoabdominal aortic aneurysm: a possible new tool for aortic endovascular surgery, International Journal of General Medicine, Vol. 5, 629-632,2012.

[17] E. Ferrero, M. Ferri, A. Viazzo, A. Robaldo, P. Carbonatto, A. Pecchio, A. Chiecchio, F. Nessi. Visceral Artery Aneurysms, an Experience on 32 Cases in a Single Center: Treatment From Surgery to Multilayer Stent, Annals of Vascular Surgery, Vol. 25, No. 7, 923-935,2011.

[18] E. Ferrero, L. Gibello, M. Ferri, A. Viazzo, F. Nessi. Aortic arch rupture after multiple multilayer stent treatment of a thoracoabdominal aneurysm, Journal of Vascular Surgery, Epub ahead of print 4 Nov 2013.

[19] S. Sultan, N. Hynes, M. Sultan, MFM Collaborators. When not to implant the multilayer flow modulator: lessons learned from application outside the indications for use in patients with thoracoabdominal pathologies, Journal of Endovascular Therapy, Vol. 21, No. 1, 96-112,2014.

[20] V. Flis, J. Matel, S. Breznik, N. Kobilica. Treatment of primary infected juxtarenal aortic aneurysm with the multilayer stent, Vascular \& Endovascular Surgery, Vol. 47, No. 7, 561-565,2013.

[21] M.M. Reijnen, S.M. van Sterkenburg. Treatment of a Salmonella-induced rapidly expanding aortic pseudoaneurysm involving the visceral arteries using the Cardiatis multilayer stent, Journal of Vascular Surgery, Epub ahead of print 11Sep 2013.

[22] A. Polydorou, M. Henry, I. Bellenis, D. Kiskinis, K. Bolos, K. Athanasiadou, A. Portinos, P. Dedeilias, I. Kokotsakis, P. Anthopoulos, G. Chondros, E. Testempasi, D. Farsaris, T. Kratimenos, C. Tsiakouri, P. Papapavlou, S. Rammos, T. Perdikides, A. Polydorou, V. Polydorou, G. Stavrou, P. Megalooikonomos, J. Moutiris, T. Fotis. Endovascular treatment of arterial aneurysms with side branches - a simple method. Myth or reality?,Hospital Chronicles,Vol. 5 No. 2, 88-94, 2010.

[23] C.C. Pieper, C. Meyer, J. Rudolph, F. Verrel, H.H. Schild, K.E Wilhelm. Interventional exclusion of iliac artery aneurysms using the flow-diverting multilayer stent, Cardiovascular \& Interventional Radiology, Vol. 36, No. 4, 917-925,2013.

[24] M. Henry, A. Polydorou, N. Frid, P. Gruffaz, A. Cavet, I. Henry, M. Hugel, D.A. Rüfenacht, L. Augsburger, M. De Beule, P. Verdonck, M. Bonneau, C. Kang, R. Ouared, B. Chopard. Treatment of renal artery aneurysm with the Multilayer Stent, Journal of Endovascular Therapy, Vol. 15, No. 2,231-236,2008. 
[25] A. Balderi, A. Antonietti, F. Pedrazzini, D. Sortino, C. Vinay, M. Grosso. Treatment of visceral aneurysm using multilayer stent: two-year follow-up results in five consecutive patients, Cardiovascular \& Interventional Radiology, Vol. 36, No. 5, 1256-61,2013.

[26] A. Balderi, A. Antonietti, F. Pedrazzini, L. Ferro, L. Leotta, E. Peano, M. Grosso. Treatment of a hepatic artery aneurysm by endovascular exclusion using the multilayer cardiatis stent, Cardiovascular \& Interventional Radiology, Vol. 33, No. 6, 1282-1286,2010.

[27] M. Natrella, M. Castagnola, F. Navarretta, M. Cristoferi, G. Fanelli, T. Meloni, F. Peinetti. Treatment of juxtarenal aortic aneurysm with the Multilayer stent, Journal Endovascular Therapy, Vol. 19, No. 1, 121-124,2012.

[28] M. Ruffino, C. Rabbia, Italian Cardiatis Registry Investigators Group. Endovascular treatment of visceral artery aneurysms with Cardiatis multilayer flow modulator: preliminary results at six-month follow-up, Journal of Cardiovascular Surgery (Torino),Vol. 52, No. 3, 311-321, 2011.
[29] E. Ferrero, M. Ferri, A. Viazzo, F. Nessi. Endovascular treatment of hepatic artery aneurysm by multilayer stents: two cases and one-year follow-up, Interactive Cardiovascular \& Thoracic Surgery, Vol. 13, No. 5, 545-547, 2011.

[30] G. Carrafiello, N. Rivolta, M. Annoni, F. Fontana, G. Piffaretti. Endovascular repair of a celiac trunk aneurysm with a new multilayer stent, Journal of Vascular Surgery, Vol. 54, No. 4, 1148-1150, 2011.

[31] C. Meyer, F. Verrel, G. Weyer, K. Wilhelm. Endovascular management of complex renal artery aneurysms using the multilayer stent, Cardiovascular\&Interventionl Radiology,Vol. 34, No. 3, 637-641,2011.

[32] DG. Hackam, DA. Redelmeier. Translation of research evidence from animals to humans,Journal of the American Medical Association, Vol. 296, No. 14, 1731-1732,2006.

[33] SM. Williams, JL. Haines, JH. Moore. The use of animal models in the study of complex disease: all else is never equal or why do so many human studies fail to replicate animal findings?,Bioessays, Vol. 26, No. 2, 170-179, 2004 